\title{
Key Derivation without Entropy Waste
}

\author{
Yevgeniy Dodis ${ }^{1, \star}$, Krzysztof Pietrzak $^{2, \star \star}$, and Daniel Wichs ${ }^{3, \star \star \star}$ \\ 1 New York University \\ ${ }^{2}$ IST Austria \\ 3 Northeastern University
}

\begin{abstract}
We revisit the classical problem of converting an imperfect source of randomness into a usable cryptographic key. Assume that we have some cryptographic application $P$ that expects a uniformly random $m$-bit key $R$ and ensures that the best attack (in some complexity class) against $P(R)$ has success probability at most $\delta$. Our goal is to design a key-derivation function (KDF) $h$ that converts any random source $X$ of min-entropy $k$ into a sufficiently "good" key $h(X)$, guaranteeing that $P(h(X))$ has comparable security $\delta^{\prime}$ which is 'close' to $\delta$.

Seeded randomness extractors provide a generic way to solve this problem for all applications $P$, with resulting security $\delta^{\prime}=O(\delta)$, provided that we start with entropy $k \geq m+2 \log (1 / \delta)-O(1)$. By a result of Radhakrishnan and Ta-Shma, this bound on $k$ (called the "RT-bound") is also known to be tight in general. Unfortunately, in many situations the loss of $2 \log (1 / \delta)$ bits of entropy is unacceptable. This motivates the study KDFs with less entropy waste by placing some restrictions on the source $X$ or the application $P$.

In this work we obtain the following new positive and negative results in this regard:

- Efficient samplability of the source $X$ does not help beat the RTbound for general applications. This resolves the SRT (samplable RT) conjecture of Dachman-Soled et al. DGKM12] in the affirmative, and also shows that the existence of computationally-secure extractors beating the RT-bound implies the existence of one-way functions.

- We continue in the line of work initiated by Barak et al. $\mathrm{BDK}^{+} 11$ and construct new information-theoretic KDFs which beat the RTbound for large but restricted classes of applications. Specifically, we design efficient KDFs that work for all unpredictability applications $P$ (e.g., signatures, MACs, one-way functions, etc.) and can either: (1) extract all of the entropy $k=m$ with a very modest security loss $\delta^{\prime}=O(\delta \cdot \log (1 / \delta))$, or alternatively, (2) achieve essentially optimal security $\delta^{\prime}=O(\delta)$ with a very modest entropy loss $k \geq m+$ $\log \log (1 / \delta)$. In comparison, the best prior results from $\mathrm{BDK}^{+} 11$ for this class of applications would only guarantee $\delta^{\prime}=O(\sqrt{\delta})$ when $k=m$, and would need $k \geq m+\log (1 / \delta)$ to get $\delta^{\prime}=O(\delta)$.
\end{abstract}

\footnotetext{
* Research partially supported by gifts from VMware Labs and Google, and NSF grants 1319051, 1314568, 1065288, 1017471, 0845003.

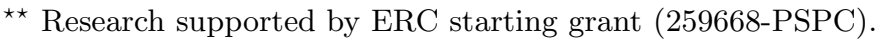

$\star \star \star$ Research supported by NSF grant 1314722 .
} 
- The weaker bounds of $\mathrm{BDK}^{+} 11$ hold for a larger class of so-called "square-friendly" applications (which includes all unpredictability, but also some important indistinguishability, applications). Unfortunately, we show that these weaker bounds are tight for the larger class of applications.

- We abstract out a clean, information-theoretic notion of $\left(k, \delta, \delta^{\prime}\right)$ unpredictability extractors, which guarantee "induced" security $\delta^{\prime}$ for any $\delta$-secure unpredictability application $P$, and characterize the parameters achievable for such unpredictability extractors. Of independent interest, we also relate this notion to the previously-known notion of (min-entropy) condensers, and improve the state-of-the-art parameters for such condensers.

\section{Introduction}

Key Derivation is a fundamental cryptographic task arising in a wide variety of situations where a given application $P$ was designed to work with a uniform $m$-bit key $R$, but in reality one only has a "weak" $n$-bit random source $X$. Examples of such sources include biometric data [DORS08, $\mathrm{BDK}^{+} 05$, physical sources [BST03, BH05], secrets with partial leakage, and group elements from Diffie-Hellman key exchange GKR04, Kra10, to name a few. We'd like to have a Key Derivation Function (KDF) $h:\{0,1\}^{n} \rightarrow\{0,1\}^{m}$ with the property that the derived key $h(X)$ can be safely used by $P$, even though the original security of $P$ was only analyzed under the assumption that its key $R$ is uniformly random.

Of course, good key derivation is generally impossible unless $X$ has some amount of entropy $k$ to begin with, where the "right" notion of entropy in this setting is min-entropy: a source $X$ has min-entropy $\mathbf{H}_{\infty}(X)=k$ if for any $x \in\{0,1\}^{n}$ we must have $\operatorname{Pr}[X=x] \leq 2^{-k}$. We call such a distribution $X$ over $n$-bits strings an $(n, k)$-source, and generally wish to design a KDF $h$ which "works" for all such $(n, k)$-sources $X$. More formally, assuming $P$ was $\delta$-secure (against some class of attackers) with the uniform key $R \equiv U_{m}$, we would like to conclude that $P$ is still $\delta^{\prime}$-secure (against nearly the same class of attackers) when using $R=h(X)$ instead. The two most important parameters are: (1) ensuring that the new security $\delta^{\prime}$ is "as close as possible" to the original security $\delta$, and (2) allowing the source entropy $k$ to be "as close as possible" to the application's key length $m$. Minimizing this threshold $k$ is very important in many practical situations. For example, in the setting of biometrics and physical randomness, many natural sources are believed to have very limited entropy, while in the setting of Diffie-Hellman key exchange reducing the size of the Diffie-Hellman group (which is roughly $2^{k}$ ) results in substantial efficiency improvements. Additionally, we prefer to achieve information-theoretic security for our KDFs (we discuss "computational KDFs" in Section 1.2), so that the derived key can be used for arbitrary (information-theoretic and computational) applications $P$.

This discussion leads us to the following central question of our work: Can one find reasonable application scenarios where one can design a provably-secure, 
information-theoretic KDF achieving "real security" $\delta^{\prime} \approx \delta$ when $k \approx m$ ? More precisely, for a given (class of) application(s) $P$,

(A) What is the best (provably) achievable security $\delta^{\prime}$ (call it $\delta^{*}$ ) when $k=m$ ?

(B) What is the smallest (provable) entropy threshold $k$ (call it $k^{*}$ ) to achieve security $\delta^{\prime}=O(\delta)$ ?

Ideally, we would like to get $\delta^{*}=\delta$ and $k^{*}=m$, and the question is how close one can come to these "ideal" bounds. In this work we will provide several positive and negative answers to our main question, including a general way to nearly achieve the above "ideal" for all unpredictability applications. But first we turn to what is known in the theory of key derivation.

RANDOMnESS ExTRACTORS. In theory, the cleanest way to design a general, information-theoretically secure KDF is by using so called (strong) randomness extractors NZ96]. Such a $(k, \varepsilon)$-extractor Ext has the property that the output distribution $\operatorname{Ext}(X)$ is $\varepsilon$-statistically close to the uniform distribution $U_{m}$, which means that using $\operatorname{Ext}(X)$ as a key will degrade the original security $\delta$ of any application $P$ by at most $\varepsilon: \delta^{\prime} \leq \delta+\varepsilon$. However, the sound use of randomness extractors comes with two important caveats. The first caveat comes from the fact that no deterministic extractor Ext can work for all $(n, k)$-sources CG89. when $k<n$, which means that extractors must be probabilistic, or "seeded". This by itself is not a big limitation, since the extracted randomness $\operatorname{Ext}(X ; S)$ is $\varepsilon$-close to $U_{m}$ even conditioned on the seed $S$, which means that the seed $S$ can be reused and globally shared across many applications 1 From our perspective, though, a more important limitation/caveat of randomness extractors comes from a non-trivial tradeoff between the min-entropy $k$ and the security $\varepsilon$ one can achieve to derive an $m$-bit $\operatorname{key} \operatorname{Ext}(X ; S)$. The best randomness extractors, such as the one given by the famous Leftover Hash Lemma (LHL) HILL99, can only achieve security $\varepsilon=\sqrt{2^{m-k}}$. This gives the following very general bound on $\delta^{\prime}$ for all applications $P$ :

$$
\delta^{\prime} \leq \delta_{\mathrm{ALL}} \stackrel{\text { def }}{=} \delta+\sqrt{2^{m-k}}
$$

Translating this bound to answer our main questions (A) and (B) above, we see that $\delta^{*}=1$ (no meaningful security is achieved when $k=m$ ) and min-entropy $k^{*} \geq m+2 \log (1 / \delta)-O(1)$ is required to get $\delta^{\prime}=O(\delta)$. For example, to derive a 128 -bit key for a CBC-MAC with security $\delta \approx \delta^{\prime} \approx 2^{-64}$, one needs $k \approx 256$ bits of min-entropy, and nothing is theoretically guaranteed when $k=128$.

Of course, part of the reason why these provable bounds are "not too great" (compared both with the "ideal" bounds, as well as the "real" bounds we will

\footnotetext{
${ }^{1}$ However, it does come with an important assumption that the source distribution $X$ must be independent of the seed $S$. Although this assumption could be problematic in some situations, such as leakage-resilient cryptography (and has led to some interesting research [TV00, $\mathrm{CDH}^{+} 00$, KZ03, DRV12]), in many situations, such as the Diffie-Hellman key exchange or biometrics, the independence of the source and the seed could be naturally enforced/assumed.
} 
achieve shortly) is their generality: extractors work for all $(n, k)$-sources $X$ and all applications $P$. Unfortunately, Radhakrishnan and Ta-shma [RTS00] showed that in this level of generality nothing better is possible: any $(k, \varepsilon)$-extractor must have $k \geq m+2 \log (1 / \varepsilon)$ (we will refer to this as the "RT-bound"). This implies that for any candidate $m$-bit extractor Ext there exists some application $P$, some (possibly inefficiently samplable) source $X$ of min-entropy $k$ and some (possibly exponential time) attacker $A$, such that $A(S)$ can break $P$ keyed by $R=\operatorname{Ext}(X ; S)$ with advantage $\sqrt{2^{m-k}}$.

Thus, there is hope that better results are possible if one restricts the type of applications $P$ (e.g., unpredictability applications), sources $X$ (e.g., efficiently samplable) or attackers $A$ (e.g., polynomial-time) considered. We discuss such options below, stating what was known together with our new results.

\subsection{Our Main Results}

Efficiently Samplable Sources. One natural restriction is to require that the source $X$ is efficiently sampleable. This restriction is known to be useful for relaxing the assumption that the source distribution $X$ is independent of the seed $S$ [TV00, DRV12, which was the first caveat in using randomness extractors. Unfortunately, it was not clear if efficient samplability of $X$ helps with reducing the entropy $\operatorname{loss} L=k-m$ below $2 \log (1 / \varepsilon)$. In fact, DachmanSoled et al. DGKM12 conjectured that this is indeed not the case when Ext is also efficient, naming this conjecture the "SRT assumption" (where SRT stands for "samplable RT").

SRT Assumption DGKM12] : For any efficient extractor Ext with $m$-bit output there exists an efficiently samplable (polynomial in $n$ ) distribution $X$ of minentropy $k=m+2 \log (1 / \varepsilon)-O(1)$ and a (generally inefficient) distinguisher $D$ which has at least an $\varepsilon$-advantage in distinguishing $(S, R=\operatorname{Ext}(X ; S))$ from $\left(S, R=U_{m}\right)$.

As our first result, we show that the SRT assumption is indeed (unfortunately) true, even without restricting the extractor Ext to be efficient.

Theorem 1. (Informal) The SRT assumption is true for any (possibly inefficient) extractor Ext. Thus, efficiently samplability does not help to reduce the entropy loss of extractors below $2 \log (1 / \varepsilon)$.

Square-Friendly Applications. The next natural restriction is to limit the class of applications $P$ in question. Perhaps, for some such applications, one can argue that the derived key $R=h_{s}(X)$ is still "good enough" for $P$ despite not being statistically close to $U_{m}$ (given $s$ ). This approach was recently pioneered by Barak et al $\mathrm{BDK}^{+} 11$, and then further extended and generalized by Dodis et al. DRV12, DY13. In these works the authors defined a special class of cryptographic applications, called square-friendly, where the pessimistic RT-bound can be provably improved. Intuitively, while any traditional application $P$ demands that the expectation (over the uniform distribution $r \leftarrow U_{m}$ ) 
of the attacker's advantage $f(r)$ on key $r$ is at most $\delta$, square-friendly applications additionally require that the expected value of $f(r)^{2}$ is also bounded by $\delta$. The works of $\mathrm{BDK}^{+} 11$, DY13 then showed that the class of square-friendly applications includes all unpredictability applications (signatures, MACs, one-way functions, etc.), and some, but not all, indistinguishability applications (including chosen plaintext attack secure encryption, weak pseudorandom functions and others). 2 Additionally, for all such square-friendly applications $P$, it was shown that universal (and thus also the stronger pairwise independent) hash functions $\left\{h_{s}\right\}$ yield the following improved bound on the security $\delta^{\prime}$ of the derived key $R=h_{s}(X)$ :

$$
\delta^{\prime} \leq \delta \mathrm{SQF} \stackrel{\text { def }}{=} \delta+\sqrt{\delta \cdot 2^{m-k}}
$$

This provable (and still relatively general!) bound lies somewhere in between the "ideal" bounds and the fully generic bound (1): in particular, for the first time we get a meaningful security $\delta^{*} \approx \sqrt{\delta}$ when $k=m$ (giving non-trivial answer to Question (A)), or, alternatively, we get full security $\delta^{\prime}=O(\delta)$ provided $k^{*} \geq m+\log (1 / \delta)$ (giving much improved answer to Question (B) than the bound $k^{*} \geq k+2 \log (1 / \delta)$ derived by using standard extractors). For example, to derive a 128 -bit key for a CBC-MAC having ideal security $\delta=2^{-64}$, we can either settle for much lower security $\delta^{\prime} \approx 2^{-32}$ from entropy $k=128$, or get full security $\delta^{\prime} \approx 2^{-64}$ from entropy $k=192$.

Given these non-trivial improvements, one can wonder if further improvements (for square-friendly applications) are still possible. As a simple (negative) result, we show that the bound in Equation (2) cannot be improved in general for all square-friendly applications. Interestingly, the proof of this result uses the proof of Theorem 1 to produce the desired source $X$ for the counter-example. For space reasons, the proof of Theorem 2 below is only given in the full version DPW13] of this paper.

Theorem 2. (Informal) There exists a $\delta$-square friendly application $P$ with an $m$-bit key such that for any family $\mathcal{H}=\left\{h_{s}\right\}$ of $m$-bit key derivation functions there exists (even efficiently samplable) $(n, k)$-source $X$ and a (generally inefficient) distinguisher $D$ such that $D(S)$ has at least $\delta^{\prime}=\Omega\left(\sqrt{\delta \cdot 2^{m-k}}\right)$ advantage in breaking $P$ with the derived key $R=h_{S}(X)$ (for random seed $S$ ).

Hence, to improve the parameters in Equation (2) and still have informationtheoretic security, we must place more restrictions on the class of applications $P$ we consider.

UnPREDiCtABILITy Applications. This brings us to our main (positive) result: we get improved information-theoretic key derivation for all unpredictability applications (which includes MACs, signatures, one-way functions, identification schemes, etc.; see Footnote 2).

\footnotetext{
${ }^{2}$ Recall, in indistinguishability applications the goal of the attack is to win a game with probability noticeably greater than $1 / 2$; in contrast, for unpredictability applications the goal of the attacker is to win with only non-negligible probability.
} 
Theorem 3. (Main Result; Informal) Assume $P$ is any unpredictability application which is $\delta$-secure with a uniform $m$-bit key against some class of attackers $\mathcal{C}$. Then, there is an efficient family of hash functions $\mathcal{H}=\left\{h_{s}:\{0,1\}^{n} \rightarrow\right.$ $\left.\{0,1\}^{m}\right\}$, such that for any $(n, k)$-source $X$, the application $P$ with the derived key $R=h_{S}(X)$ (for random public seed $S$ ) is $\delta^{\prime}$-secure against class $\mathcal{C}$, where:

$$
\delta^{\prime}=O\left(1+\log (1 / \delta) \cdot 2^{m-k}\right) \delta .
$$

In particular, we get the following nearly optimal answers to Questions (A), (B):

- With entropy $k=m$, we get security $\delta^{*}=(1+\log (1 / \delta)) \delta$ (answering Question (A)).

- To get security $\delta^{\prime} \leq 3 \delta$, we only need entropy $k^{*}=m+\log \log (1 / \delta)+4$ (answering Question (B)).

In fact, our basic KDF hash family $\mathcal{H}$ is simply a $t$-wise independent hash function where $t=O(\log (1 / \delta))$. Hence, by using higher than pairwise independence (which was enough for weaker security given by Equations (11) and (2)), we get a largely improved entropy loss: $\log \log (1 / \delta)$ instead of $\log (1 / \delta)$.

As we can see, the provable bounds above nearly match the ideal bounds $\delta^{*}=\delta$ and $k^{*}=m$ and provide a vast improvement over what was known previously. For example, to derive a 128-bit key for a CBC-MAC having ideal security $\delta=2^{-64}$ (so that $\log \log (1 / \delta)=6$ ), we can either have excellent security $\delta^{\prime} \leq 2^{-57.9}$ starting with minimal entropy $k=128$, or get essentially full security $\delta^{\prime} \leq 2^{-62.4}$ with only slightly higher entropy $k=138$. Thus, for the first time we obtained an efficient, theoretically-sound key derivation scheme which nearly matches "dream" parameters $k^{*}=m$ and $\delta^{*}=\delta$. Alternatively, as we discuss in Section 1.2 for the first time we can offer a provably-secure alternative to the existing practice of using cryptographic hash functions modeled as a random oracle for KDFs, and achieve nearly optimal parameters.

Unpredictability Extractors and Condensers. To better understand the proof of Theorem 3 , it is helpful to abstract the notion of an unpredictability extractor UExt which we define in this work. Recall, standard $(k, \varepsilon)$-extractors $\varepsilon$ fool any distinguisher $D(R, S)$ trying to distinguish $R=\operatorname{Ext}(X ; S)$ from $R$ being uniform. In contrast, when dealing with $\delta$-secure unpredictability applications, we only care about "fooling" so called $\delta$-distinguishers $D$ : these are distinguishers s.t. $\operatorname{Pr}\left[D\left(U_{m}, S\right)=1\right] \leq \delta$, which directly corresponds to the emulation of $P$ 's security experiment between the "actual attacker" $A$ and the challenger $C$. Thus, we define $\left(k, \delta, \delta^{\prime}\right)$-unpredictability extractors as having the property that $\operatorname{Pr}[D(\operatorname{UExt}(X ; S), S)=1] \leq \delta^{\prime}$ for any $\delta$-distinguisher $D 3$ With this cleaner notion in mind, our main Theorem 3 can be equivalently restated as follows:

Theorem 4. (Main Result; Restated) A family $\mathcal{H}=\left\{h_{s}:\{0,1\}^{n} \rightarrow\{0,1\}^{m}\right\}$ which is $O(\log (1 / \delta))$-wise independent defines $a\left(k, \delta, O\left(1+\log (1 / \delta) \cdot 2^{m-k}\right) \delta\right)$ unpredictability extractor $\operatorname{UExt}(x ; s)=h_{s}(x)$.

\footnotetext{
${ }^{3}$ This notion can also be viewed as "one-sided" slice extractors RTS00. Unlike this work, though, the authors of [RTS00] did not use slice extractors as an interesting primitive by itself, and did not offer any constructions of such extractors.
} 
In turn, we observe that unpredictability extractors are closely connected to the related notion of a randomness condenser [RR99, RSW06]: such a $(k, \ell, \varepsilon)$ condenser Cond : $\{0,1\}^{n} \rightarrow\{0,1\}^{m}$ has the property that the output distribution Cond $(X ; S)$ is $\varepsilon$-close (even given the seed $S$ ) to some distribution $Y$ s.t. the conditional min-entropy $\mathbf{H}_{\infty}(Y \mid S) \geq m-\ell$ whenever $\mathbf{H}_{\infty}(X) \geq k$. In particular, instead of requiring the output to be close to uniform, we require it to be close to having almost full entropy, with some small "gap" $\ell$. While $\ell=0$ gives back the definition of $(k, \varepsilon)$-extractors, permitting a small non-zero "entropy gap" $\ell$ has recently found important applications for key derivation $\mathrm{BDK}^{+} 11$, DRV12, DY13. In particular, it is easy to see that a $(k, \ell, \varepsilon)$-condenser is also a $\left(k, \delta, \varepsilon+\delta \cdot 2^{\ell}\right)$-unpredictability extractor. Thus, to show Theorem 4 it suffices to show that $O(\log (1 / \delta))$-wise independent hashing gives a $(k, \ell, \delta)$-condenser, where $\ell \approx \log \log (1 / \delta)$.

Theorem 5. (Informal) $A$ family $\mathcal{H}=\left\{h_{s}:\{0,1\}^{n} \rightarrow\{0,1\}^{m}\right\}$ of $O(\log (1 / \delta)$ )wise independent hash functions defines $a(k, \ell, \delta)$-condenser $\operatorname{Cond}(x ; s)=h_{s}(x)$ for either of the following settings:

- No Entropy Loss: min-entropy $k=m$ and entropy gap $\ell=\log \log (1 / \delta)$.

- Constant Entropy Gap: min-entropy $k=m+\log \log (1 / \delta)+O(1)$ and entropy gap $\ell=1$.

It is instructive to compare this result with the RT-bound for $(k, \delta)$-extractors: to have no entropy gap $\ell=0$ requires us to start with entropy $k \geq m+2 \log (1 / \delta)$. However, already 1-bit entropy gap $\ell=1$ allows us to get away with $k=$ $m+\log \log (1 / \delta)$, while further increasing the gap to $\ell=\log \log (1 / \delta)$ results in no entropy loss $k=m$.

Balls and Bins, MaX-LoAd And Balanced Hashing. Finally, to prove Theorem 5 (and, thus, Theorem 4 and Theorem 3) we further reduce the problem of condensers to a very simple balls-and-bins problem. Indeed, we can think of our $(k, \ell, \delta)$-condenser as a way to hash $2^{k}$ items (out of a universe of size $2^{n}$ ) into $2^{m}$ bins, so that the load (number of items per bin) is not too much larger than the expected $2^{k-m}$ for "most" of the bins. More concretely, it boils down to analyzing a version of average-load: if we choose a random item (and a random hash function from the family) then the probability that the item lands in a bin with more than $2^{\ell}\left(2^{k-m}\right)$ items should be at most $\varepsilon$. We use Chernoff-type bounds for limited independence [Sie89, BR94] to analyze this version of average load when the hash function is $O(\log 1 / \delta)$-independent.

Optimizing Seed Length. The description length $d$ of our $O(\log (1 / \delta))$-wise independent $\mathrm{KDF} h_{s}$ is $d=O(n \log (1 / \delta))$ bits, which is much larger than that needed by universal hashing for standard extractors. In the full version of this paper DPW13, we show how to adapt the elegant "gradual increase of independence" technique of Celis et al. CRSW11 to reduce the seed length to nearly linear: $d=O(n \log k)$ (e.g., for $k=128$ and $\delta=2^{-64}$ this reduces the seed length from $128 n$ to roughly $7 n$ bits). It is an interesting open problem if the seed length can be reduced even further (and we show non-constructively that the answer is positive). 


\subsection{Computational Extractors}

So far we considered information-theoretic techniques for designing theoreticallysound KDFs. Of course, given the importance of the problem, it is also natural to see if better parameters can be obtained when we assume that the attacker $A$ is computationally bounded. We restrict our attention to the study of computational extractors $\left[\mathrm{DGH}^{+} 04\right.$, Kra10, DGKM12] Ext, whose output $R=\operatorname{Ext}(X ; S)$ looks pseudorandom to $D$ (given $S$ ) for any efficiently samplable $(n, k)$-source $X$, which would suffice for our KDF goals if very strong results were possible for such extractors.

Unfortunately, while not ruling out the usefulness of computational extractors, we point out the following three negative results: (1) even "heuristic" computational computational extractors do not appear to beat the information-theoretic bound $k^{*} \geq m$ (which we managed to nearly match for all unpredictability applications); (2) existing "provably-secure" computational extractors do not appear to offer any improvement to our information-theoretic KDFs, when dealing with the most challenging "low entropy regime" (when $k$ is roughly equal to the security parameter); (3) even for "medium-to-high entropy regimes", computational extractors beating the RT-bound require one-way functions. We discuss these points in the full version DPW13, here only briefly mentioning points (1) and (3).

Heuristic Extractors. In practice, one would typically use so called "cryptographic hash function" $h$, such as SHA or MD5, for key derivation (or as a computational extractor). As discussed in detail by [ $\mathrm{DGH}^{+} 04$, Kra10, DRV12, there are several important reasons for this choice. From the perspective of this work, we will focus on the arguably the most important such reason - the common belief that cryptographic hash functions achieve excellent security $\delta^{\prime} \approx \delta$ already when $k \approx m$. This can be easily justified in the random oracle model; assuming the $\mathrm{KDF} h$ is a random oracle which can be evaluated on at most $q$ points (where $q$ is the upper bound of the attacker's running time), one can upper bound $\delta^{\prime} \leq \delta+q / 2^{k}$, where $q / 2^{k}$ is the probability the attacker evaluates $h(X)$. In turn, for most natural computationally-secure applications, in time $q$ the attacker can also test about $q$ out of $2^{m}$ possible $m$-bit keys, and hence achieve advantage $q / 2^{m}$. This means that the ideal security $\delta$ of $P$ cannot be lower than $q / 2^{m}$, implying $q \leq \delta \cdot 2^{m}$. Plugging this bound on $q$ in the bound of $\delta^{\prime} \leq \delta+q / 2^{k}$ above, we get that using a random oracle (RO) as a computational extractor/KDF achieves real security

$$
\delta^{\prime} \leq \delta_{\mathrm{RO}} \stackrel{\text { def }}{=} \delta+\delta \cdot 2^{m-k}
$$

Although this heuristic bound is indeed quite amazing (e.g., $\delta^{\prime} \leq 2 \delta$ even when $k=m$, meaning that $\delta^{*}=2 \delta$ and $k^{*}=m$ ), and, unsurprisingly, beats our provably-secure, information-theoretic bounds, it still requires $k^{*} \geq m$. So we 
are not that far off, especially given our nearly matching bound for all unpredictability applications 4

BeAting RT-Bound Implies OWFs. As observed by Kra10, DGKM12, for medium-to-high entropy regimes, computational assumptions help in "beating" the RT-bound $k \geq m+2 \log (1 / \varepsilon)$ for any $(k, \varepsilon)$-secure extractor, as applying the PRG allows one to increase $m$ essentially arbitrarily (while keeping the original min-entropy the same). Motivated by this, Dachman-Soled et al. DGKM12] asked an interesting theoretical question if the existence of one-way functions (and, hence, PRGs HILL99) is essential for beating the RT-bound for unconditional extractors. They also managed to give an affirmative answer to this question under the SRT assumption mentioned earlier. Since we unconditionally prove the SRT assumption (see Theorem 1), we immediately get the following Corollary, removing the conditional clause from the result of [DGKM12]:

Theorem 6. (Informal) If Ext is an efficient $(k, \varepsilon)$-computational extractor with an $m$-bit output, where $m>k-2 \log (1 / \varepsilon)-O(1)$, then one-way functions (and, hence, PRGs) exist.

\section{Preliminaries}

We recap some definitions and results from probability theory. Let $X, Y$ be random variables with supports $S_{X}, S_{Y}$, respectively. We define their statistical difference as

$$
\Delta(X, Y)=\frac{1}{2} \sum_{u \in S_{X} \cup S_{Y}}|\operatorname{Pr}[X=u]-\operatorname{Pr}[Y=u]| .
$$

We write $X \approx_{\varepsilon} Y$ and say that $X$ and $Y$ are $\varepsilon$-statistically close to denote that $\Delta(X, Y) \leq \varepsilon$.

The min-entropy of a random variable $X$ is $\mathbf{H}_{\infty}(X) \stackrel{\text { def }}{=}-\log \left(\max _{x} \operatorname{Pr}[X=\right.$ $x]$ ), and measures the "best guess" for $X$. The conditional min-entropy is defined by $\mathbf{H}_{\infty}(X \mid Y=y) \stackrel{\text { def }}{=}-\log \left(\max _{x} \operatorname{Pr}[X=x \mid Y=y]\right)$. Following Dodis et al. DORS08, we define the average conditional min-entropy:

$$
\begin{aligned}
\mathbf{H}_{\infty}(X \mid Y) & \stackrel{\text { def }}{=}-\log \left(\underset{y \leftarrow Y}{\mathbb{E}}\left[\max _{x} \operatorname{Pr}[X=x \mid Y=y]\right]\right) \\
& =-\log \left(\underset{y \leftarrow Y}{\mathbb{E}}\left[2^{-\mathbf{H}_{\infty}(X \mid Y=y)}\right]\right) .
\end{aligned}
$$

Above, and throughout the paper, all "log" terms are base 2 , unless indicated otherwise. We say that a random variable $X$ is an $(n, k)$-source if the support of $X$ is $\{0,1\}^{n}$ and the entropy of $X$ is $\mathbf{H}_{\infty}(X) \geq k$.

\footnotetext{
${ }^{4}$ Also, unlike our bound in Equation (3), one cannot apply the heuristic bound from Equation (4) to derive a key for an information-theoretically secure MAC.
} 
Lemma 1 (A Tail Inequality [BR94]). Let $q \geq 4$ be an even integer. Suppose $X_{1}, \ldots, X_{n}$ are $q$-wise independent random variables taking values in $[0,1]$. Let $X:=X_{1}+\cdots+X_{n}$ and define $\mu:=\mathbf{E}[X]$ be the expectation of the sum. Then, for any $A>0, \operatorname{Pr}[|X-\mu| \geq A] \leq 8\left(\frac{q \mu+q^{2}}{A^{2}}\right)^{q / 2}$. In particular, for any $\alpha>0$ and $\mu>q$, we have $\operatorname{Pr}[X \geq(1+\alpha) \mu] \leq 8\left(\frac{2 q}{\alpha^{2} \mu}\right)^{q / 2}$.

\section{Defining Extractors for Unpredictability Applications}

We start by abstracting out the notion of general unpredictability applications (e.g., one-way functions, signatures, message authentication codes, soundness of an argument, etc.) as follows. The security of such all such primitives is abstractly defined via a security game $P$ which requires that, for all attackers $\mathcal{A}$ (in some complexity class), $\operatorname{Pr}\left[P^{\mathcal{A}}(U)=1\right] \leq \delta$ where $P^{\mathcal{A}}(U)$ denotes the execution of the game $P$ with the attacker $\mathcal{A}$, where $P$ uses the uniform randomness $U$ 5 For example, in the case of a message-authentication code (MAC), the value $U$ is used as secret key for the MAC scheme and the game $P$ is the standard "existential unforgeability against chosen-message attack game" for the given MAC. Next, we will assume that $\delta$ is some small (e.g., negligible) value, and ask the question if we can still use the primitive $P$ if, instead of a uniformly random $U$, we only have some arbitrary $(n, k)$-source $X$ ?

To formally answer this question, we would like a function UExt : $\{0,1\}^{n} \times$ $\{0,1\}^{d} \rightarrow\{0,1\}^{m}$ (seeded unpredictability extractor) such that, for all attackers $\mathcal{A}$ (in some complexity class), $\operatorname{Pr}\left[P^{\mathcal{A}(S)}(\operatorname{UExt}(X ; S))=1\right] \leq \varepsilon$, where the seed $S$ is chosen uniformly at random and given to the attacker, and $\varepsilon$ is not much larger than $\delta$. Since we do not wish to assume much about the application $P$ or the attacker $\mathcal{A}$, we can roll them up into a unified adversarial "distinguisher" defined by $D(R, S):=P^{\mathcal{A}(S)}(R)$. By definition, if $R=U$ is random and independent of $S$, then $\operatorname{Pr}[D(U, S)=1]=\operatorname{Pr}\left[P^{\mathcal{A}(S)}(U)=1\right] \leq \delta$. On the other hand, we need to ensure that $\operatorname{Pr}\left[P^{\mathcal{A}(S)}(\operatorname{UExt}(X ; S))=1\right]=\operatorname{Pr}[D(\operatorname{UExt}(X ; S), S)=1] \leq$ $\varepsilon$ for some $\varepsilon$ which is not much larger than $\delta$. This motivates the following definition of unpredictability extractor which ensures that the above holds for all distinguishers $D$.

Definition 1 (UExtract). We say that a function $D:\{0,1\}^{m} \times\{0,1\}^{d} \rightarrow$ $\{0,1\}$ is a $\delta$-distinguisher if $\operatorname{Pr}[D(U, S)=1] \leq \delta$ where $(U, S)$ is uniform over $\{0,1\}^{m} \times\{0,1\}^{d}$. A function UExt : $\{0,1\}^{n} \times\{0,1\}^{d} \rightarrow\{0,1\}^{m}$ is a $(k, \delta, \varepsilon)$-unpredictability extractor (UExtract) if for any $(n, k)$-source $X$ and any $\delta$-distinguisher $D$, we have $\operatorname{Pr}[D(\operatorname{UExt}(X ; S), S)=1] \leq \varepsilon$ where $S$ is uniform over $\{0,1\}^{d}$.

${ }^{5}$ In contrast, for indistinguishability games we typically require that $\operatorname{Pr}\left[P^{\mathcal{A}}(U)=\right.$ $1] \leq \frac{1}{2}+\delta$. 
Notice that the above definition is essentially the same as that of standard extractors except that: (1) we require that the distinguisher has a "small" probability $\delta$ of outputting 1 on the uniform distribution, and (2) we only require a one-sided error that the probability of outputting 1 does not increase too much. A similar notion was also proposed by [RTS00] and called a "slice extractor".

Toward the goal of understanding unpredictability extractors, we show tight connections between the above definition and two seemingly unrelated notions. Firstly, we define "condensers for min-entropy" and show that the they yield "good" unpredictability extractors. Second, we define something called "balanced hash functions" and show that they yield good condensers, and therefore also good unpredictability extractors. Lastly, we show that unpredictability extractors also yield balanced hash functions, meaning that all three notions are essentially equivalent up to a small gap in parameters.

Definition 2 (Condenser). A function Cond : $\{0,1\}^{n} \times\{0,1\}^{d} \rightarrow\{0,1\}^{m}$ is $a(k, \ell, \varepsilon)$-condenser if for all $(n, k)$-sources $X$, and a uniformly random and independent seed $S$ over $\{0,1\}^{d}$, the joint distribution $(S$, Cond $(X ; S))$ is $\varepsilon$ statistically-close to some joint distribution $(S, Y)$ such that, for all $s \in\{0,1\}^{d}$, $\mathbf{H}_{\infty}(Y \mid S=s) \geq m-\ell$.

First, we show that condensers already give us unpredictability extractors. This is similar in spirit to a lemma of DY13 which shows that, if we use a key with a small entropy gap for an unpredictability application, the security of the application is only reduced by at most a small amount. One difference that prevents us from using that lemma directly is that we need to explicitly include the seed of the condenser and the dependence between the condenser output and the seed.

Lemma 2 (Condenser $\Rightarrow$ UExtract). Any $(k, \ell, \varepsilon)$-condenser is a $\left(k, \delta, \varepsilon^{*}\right)$ UExtract where $\varepsilon^{*}=\varepsilon+2^{\ell} \delta$.

Proof. Let Cond : $\{0,1\}^{n} \times\{0,1\}^{d} \rightarrow\{0,1\}^{m}$ be a $(k, \ell, \varepsilon)$-condenser and let $X$ be an $(n, k)$-source. Let $S$ be uniform over $\{0,1\}^{d}$, so that, by definition, there is a joint distribution $(S, Y)$ which has statistical distance at most $\varepsilon$ from $(S$, Cond $(X ; S))$ such that $\mathbf{H}_{\infty}(Y \mid S=s) \geq m-\ell$ for all $s \in\{0,1\}^{d}$. Therefore, for any $\delta$-distinguisher $D$, we have

$$
\begin{aligned}
\operatorname{Pr}[D(\operatorname{Cond}(X ; S), S)=1] & \leq \varepsilon+\operatorname{Pr}[D(Y, S)=1] \\
& =\varepsilon+\sum_{y, s} \operatorname{Pr}[S=s] \operatorname{Pr}[Y=y \mid S=s] \operatorname{Pr}[D(y, s)=1] \\
& \leq \varepsilon+\sum_{y, s} 2^{-d} 2^{-\mathbf{H}_{\infty}(Y \mid S=s)} \operatorname{Pr}[D(y, s)=1] \\
& \leq \varepsilon+2^{\ell} \sum_{y, s} 2^{-(m+d)} \operatorname{Pr}[D(y, s)=1] \leq \varepsilon+2^{\ell} \delta .
\end{aligned}
$$

Definition 3 (Balanced Hashing). Let $h:=\left\{h_{s}:\{0,1\}^{n} \rightarrow\{0,1\}^{m}\right\}_{s \in\{0,1\}^{d}}$ be a hash function family. For $\mathcal{X} \subseteq\{0,1\}^{n}, s \in\{0,1\}^{d}, x \in \mathcal{X}$ we define 
$\operatorname{Load}_{\mathcal{X}}(x, s):=\left|\left\{x^{\prime} \in \mathcal{X}: h_{s}\left(x^{\prime}\right)=h_{s}(x)\right\}\right| 6$ We say that the family $h$ is $(k, t, \varepsilon)$ balanced if for all $\mathcal{X} \subseteq\{0,1\}^{n}$ of size $|\mathcal{X}|=2^{k}$, we have

$$
\operatorname{Pr}\left[\operatorname{Load}_{\mathcal{X}}(X, S)>t 2^{k-m}\right] \leq \varepsilon
$$

where $S, X$ are uniformly random and independent over $\{0,1\}^{d}, \mathcal{X}$ respectively.

Lemma 3 (Balanced $\Rightarrow$ Condenser). Let $\mathcal{H}:=\left\{h_{s}:\{0,1\}^{n} \rightarrow\{0,1\}^{m}\right\}_{s \in\{0,1\}^{d}}$ be $a(k, t, \varepsilon)$-balanced hash function family. Then the function Cond : $\{0,1\}^{n} \times$ $\{0,1\}^{d} \rightarrow\{0,1\}^{m}$ defined by $\operatorname{Cond}(x ; s)=h_{s}(x)$ is a $(k, \ell, \varepsilon)$-condenser for $\ell=$ $\log (t)$.

Proof. Without loss of generality, we can restrict ourselves to showing that Cond satisfies the condenser definition for every flat source $X$ which is uniformly random over some subset $\mathcal{X} \subseteq\{0,1\}^{n},|\mathcal{X}|=2^{k}$. Let us take such a source $X$ over the set $\mathcal{X}$, and define a modified hash family $\tilde{h}=\left\{\tilde{h}_{s}: \mathcal{X} \rightarrow\{0,1\}^{m}\right\}_{s \in\{0,1\}^{d}}$ which depends on $\mathcal{X}$ and essentially "re-balances" $h$ on the set $\mathcal{X}$. In particular, for every pair $(s, x)$ such that $\operatorname{Load}_{\mathcal{X}}^{h}(x, s) \leq t 2^{k-m}$ we set $\tilde{h}_{s}(x):=h_{s}(x)$, and for all other pairs $(s, x)$ we define $\tilde{h}_{s}(x)$ in such a way that $\operatorname{Load}_{\mathcal{X}}(x, s) \leq t 2^{k-m}$ (the super-script is used to denote the hash function with respect to which we are computing the load). It is easy to see that this "re-balancing" is always possible. We use the re-balanced hash function $\tilde{h}$ to define a joint distribution $(S, Y)$ by choosing $S$ uniformly at random over $\{0,1\}^{d}$, choosing $X$ uniformly/independently over $\mathcal{X}$ and setting $Y=\tilde{h}_{S}(X)$. It's easy to check that the statistical distance between $(S, \operatorname{Cond}(X ; S))$ and $(S, Y)$ is at most $\operatorname{Pr}\left[h_{S}(X) \neq \tilde{h}_{S}(X)\right] \leq \operatorname{Pr}\left[\operatorname{Load}_{\mathcal{X}}^{h}(X, S)>t 2^{k-m}\right] \leq \varepsilon$. Furthermore, for every $s \in\{0,1\}^{d}$, we have:

$$
\begin{aligned}
\mathbf{H}_{\infty}(Y \mid S=s) & =-\log \left(\max _{y} \operatorname{Pr}[Y=y \mid S=s]\right) \\
& =-\log \left(\max _{y} \operatorname{Pr}\left[X \in \tilde{h}_{s}^{-1}(y)\right]\right) \geq-\log \left(t 2^{k-m} / 2^{k}\right)=m-\log t .
\end{aligned}
$$

Therefore Cond is a $(k, \ell=\log t, \varepsilon)$-condenser.

Lemma 4 (UExtract $\Rightarrow$ Balanced). Let UExt : $\{0,1\}^{n} \times\{0,1\}^{d} \rightarrow\{0,1\}^{m}$ be $a(k, \delta, \varepsilon)$-UExtractor for some, $\varepsilon>\delta>0$. Then the hash family $\mathcal{H}=\left\{h_{s}\right.$ : $\left.\{0,1\}^{n} \rightarrow\{0,1\}^{m}\right\}_{s \in\{0,1\}^{d}}$ defined by $h_{s}(x)=\operatorname{UExt}(x ; s)$ is $(k, \varepsilon / \delta, \varepsilon)$-balanced.

Proof. Let $t=\varepsilon / \delta$ and assume that $\mathcal{H}$ is not $(k, t, \varepsilon)$-balanced. Then there exists some set $\mathcal{X} \subseteq\{0,1\}^{n},|\mathcal{X}|=2^{k}$ such that $\hat{\varepsilon}:=\operatorname{Pr}\left[\operatorname{Load}_{\mathcal{X}}(X, S)>t 2^{k-m}\right]>\varepsilon$ where $X$ is uniform over $\mathcal{X}$ and $S$ is uniform over $\{0,1\}^{d}$. Let $\mathcal{X}_{s} \subseteq \mathcal{X}$ be defined by $\mathcal{X}_{s}:=\left\{x \in \mathcal{X}: \operatorname{Load}_{\mathcal{X}}(x, s)>t 2^{k-m}\right\}$ and let $\varepsilon_{s} \stackrel{\text { def }}{=}\left|\mathcal{X}_{s}\right| / 2^{k}$. By definition $\hat{\varepsilon}=\sum_{s} 2^{-d} \varepsilon_{s}$. Define $\mathcal{Y}_{s} \subseteq\{0,1\}^{m}$ via $\mathcal{Y}_{s}:=h_{s}\left(\mathcal{X}_{s}\right)$. Now by definition, each $y \in \mathcal{Y}_{s}$ has at least $t 2^{k-m}$ pre-images in $\mathcal{X}_{s}$ and therefore $\delta_{s} \stackrel{\text { def }}{=}\left|\mathcal{Y}_{s}\right| / 2^{m} \leq$ $\left|\mathcal{X}_{s}\right| /\left(t 2^{k-m} 2^{m}\right) \leq \varepsilon_{s} / t$ and $\delta:=\sum_{s} 2^{-d} \delta_{s} \leq \hat{\varepsilon} / t$.

Define the distinguisher $D$ via $D(y, s)=1$ iff $y \in \mathcal{Y}_{s}$. Then $D$ is a $\delta$ distinguisher for $\delta \leq \hat{\varepsilon} / t \leq \varepsilon / t$ but $\operatorname{Pr}\left[D\left(h_{S}(X), S\right)=1\right]=\hat{\varepsilon} \geq \varepsilon$. Therefore, UExt is not a $(k, \varepsilon / t, \varepsilon)$-UExtractor.

\footnotetext{
${ }^{6}$ Note that we allow $x^{\prime}=x$ and so $\operatorname{Load} \mathcal{X}(x, s) \geq 1$.
} 
Summary. Taking all of the above lemmata together, we see that they are close to tight. In particular, for any $\varepsilon>\delta>0$, we get:

$$
(k, \delta, \varepsilon) \text {-UExt } \stackrel{\operatorname{Lem} 4}{\Rightarrow}(k, \varepsilon / \delta, \varepsilon) \text {-Balanced } \stackrel{\operatorname{Lem} 3}{\Rightarrow}
$$

$(k, \log (\varepsilon / \delta), \varepsilon)$-Condenser $\stackrel{\operatorname{Lem} 2}{\Rightarrow}(k, \delta, 2 \varepsilon)$-UExt

\section{Constructing Unpredictability Extractors}

Given the connections established in the previous section, we have paved the road for constructing unpredictability extractors via balanced hash functions, which is a seemingly simpler property to analyze. Indeed, we will give relatively simple lemmas showing that "sufficiently independent" hash functions are balanced. This will lead to the following parameters (restating Theorem 3 from the introduction):

Theorem 7. There exists an efficient $(k, \delta, \varepsilon)$-unpredictability extractor UExt : $\{0,1\}^{n} \times\{0,1\}^{d} \rightarrow\{0,1\}^{m}$ for the following parameters:

1. When $k=m$ (no entropy loss), we get $\varepsilon=(1+\log (1 / \delta)) \delta$.

2. When $k \geq m+\log \log 1 / \delta+4$, we get $\varepsilon=3 \delta$.

3. In general, $\varepsilon=O\left(1+2^{m-k} \log (1 / \delta)\right) \delta$.

In all cases, the function UExt is simply a $(\log (1 / \delta)+O(1))$-wise independent hash function and the seed length is $d=O(n \log (1 / \delta))$.

Although these constructions may already be practical, the level of independence we will need is $O(\log 1 / \delta)$, which will result in a large seed $O(n \log (1 / \delta))$. We will show how to achieve similar parameters with a shorter seed $O(n \log k)$ in the full version of this paper DPW13. We now proceed to prove all of the parts of Theorem 7 by constructing "good" balanced hash functions and using our connections between balanced hashing and unpredictability extractors from the previous section.

\subsection{Sufficient Independence Provides Balance}

First we start with a simple case where the output $m$ is equal to the entropy $k$.

Lemma 5. Let $\mathcal{H}:=\left\{h_{s}:\{0,1\}^{n} \rightarrow\{0,1\}^{k}\right\}_{s \in\{0,1\}^{d}}$ be $(t+1)$-wise independent. Then it is $(k, t, \varepsilon)$-balanced where $\varepsilon \leq\left(\frac{e}{t}\right)^{t}$ and $e$ is the base of the natural logarithm.

Proof. Fix any set $\mathcal{X} \subseteq\{0,1\}^{n}$ of size $|\mathcal{X}|=2^{k}$. Let $X$ be uniform over $\mathcal{X}$ and $S$ be uniform/independent over $\{0,1\}^{d}$. Then

$$
\begin{aligned}
\operatorname{Pr}\left[\operatorname{Load}_{\mathcal{X}}(X, S)>t\right] & \leq \operatorname{Pr}\left[\exists \mathcal{C} \subseteq \mathcal{X},|\mathcal{C}|=t \quad \forall x^{\prime} \in \mathcal{C}: h_{S}\left(x^{\prime}\right)=h_{S}(X) \wedge x^{\prime} \neq X\right] \\
& \leq \sum_{\mathcal{C} \subseteq \mathcal{X},|\mathcal{C}|=t} \operatorname{Pr}\left[\forall x^{\prime} \in \mathcal{C}: h_{S}\left(x^{\prime}\right)=h_{S}(X) \wedge x^{\prime} \neq X\right] \\
& \leq\left(\begin{array}{c}
2^{k} \\
t
\end{array}\right) 2^{-t k} \leq\left(\frac{e 2^{k}}{t}\right)^{t} 2^{-t k} \leq\left(\frac{e}{t}\right)^{t} .
\end{aligned}
$$


Corollary 1. For any $0<\varepsilon<2^{-2 e}$, any $\delta>0$, a $(\lceil\log (1 / \varepsilon)\rceil+1)$-wise independent hash family $\mathcal{H}=\left\{h_{s}:\{0,1\}^{n} \rightarrow\{0,1\}^{k}\right\}_{s \in\{0,1\}^{d}}$ is:

$(k, \log (1 / \varepsilon), \varepsilon)$-balanced, $\quad(k, \log \log (1 / \varepsilon), \varepsilon)$-condenser,$\quad(k, \delta, \log (1 / \varepsilon) \delta+$ $\varepsilon)$-UExtractor.

In particular, setting $\delta=\varepsilon$, it is a $(k, \delta,(1+\log (1 / \delta)) \delta)$-UExtractor.

Proof. Set $t=\lceil\log (1 / \varepsilon)\rceil$ in Lemma 5 and notice that $\left(\frac{e}{t}\right)^{t} \leq 2^{-t} \leq \varepsilon$ as long as $t \geq 2 e$.

This establishes part (1) of Theorem 17. Next we look at a more general case where $k$ may be larger than $m$. This also covers the case $k=m$ but gets a somewhat weaker bound. It also requires a more complex tail bound for $q$-wise independent variables.

Lemma 6. Let $\mathcal{H}:=\left\{h_{s}:\{0,1\}^{n} \rightarrow\{0,1\}^{m}\right\}_{s \in\{0,1\}^{d}}$ be $(q+1)$-wise independent for some even $q$. Then, for any $\alpha>0$, it is $(k, 1+\alpha, \varepsilon)$-balanced where $\varepsilon \leq 8\left(\frac{q 2^{k-m}+q^{2}}{\left(\alpha 2^{k-m}-1\right)^{2}}\right)^{q / 2}$.

Proof. Let $\mathcal{X} \subseteq\{0,1\}^{n}$ be a set of size $|\mathcal{X}|=2^{k}, X$ be uniform over $\mathcal{X}$, and $S$ be uniform/independent over $\{0,1\}^{d}$. Define the indicator random variables $C\left(x^{*}, x\right)$ to be 1 if $h_{S}(x)=h_{S}\left(x^{*}\right)$ and 0 otherwise. Then:

$$
\begin{aligned}
& \operatorname{Pr}\left[\operatorname{Load}_{\mathcal{X}}(X, S)>(1+\alpha) 2^{k-m}\right] \\
= & \sum_{x^{*} \in \mathcal{X}} \operatorname{Pr}\left[X=x^{*}\right] \operatorname{Pr}\left[\operatorname{Load}_{\mathcal{X}}\left(x^{*}, S\right)>(1+\alpha) 2^{k-m}\right] \\
= & 2^{-k} \sum_{x^{*} \in \mathcal{X}} \operatorname{Pr}\left[\sum_{x \in \mathcal{X} \backslash\left\{x^{*}\right\}} C\left(x^{*}, x\right)+1>(1+\alpha) 2^{k-m}\right] \\
\leq & 8\left(\frac{q 2^{k-m}+q^{2}}{\left(\alpha 2^{k-m}-1\right)^{2}}\right)^{q / 2}
\end{aligned}
$$

Where the last line follows from the tail inequality Lemma 1 with the random variables $\left\{C\left(x^{*}, x\right)\right\}_{x \in \mathcal{X} \backslash\left\{x^{*}\right\}}$ which are $q$-wise independent and have expected value $\mu=\mathbb{E}\left[\sum_{x \in \mathcal{X} \backslash\left\{x^{*}\right\}} C\left(x^{*}, x\right)\right]=\left(2^{k}-1\right) 2^{-m} \leq 2^{k-m}$, and by setting $A=$ $(1+\alpha) 2^{k-m}-1-\mu \geq \alpha 2^{k-m}-1$; recall that $C\left(x^{*}, x^{*}\right)$ is always 1 and $C\left(x^{*}, x\right)$ for $x \neq x^{*}$ is 1 with probability $2^{-m}$.

Corollary 2. For any $0<\varepsilon<2^{-5}, k \geq m+\log \log (1 / \varepsilon)+4, a(\lceil\log (1 / \varepsilon)\rceil+6)$ wise independent hash function family $\mathcal{H}=\left\{h_{s}:\{0,1\}^{n} \rightarrow\{0,1\}^{m}\right\}_{s \in\{0,1\}^{d}}$ is:

$(k, 2, \varepsilon)$-balanced, $\quad(k, 1, \varepsilon)$-condenser,$\quad(k, \delta, 2 \delta+\varepsilon)$-UExt for any $\delta>0$. In particular, setting $\delta=\varepsilon$, it is a $(k, \delta, 3 \delta)$-UExt. 
Proof. Set $\alpha:=1$ and choose $q \in(\log (1 / \varepsilon)+3, \log (1 / \varepsilon)+5)$ to be an even integer. Notice that $2^{k-m} \geq 16 \log (1 / \varepsilon) \geq 8(\log (1 / \varepsilon)+5) \geq 8 q$ since $\log (1 / \varepsilon) \geq 5$. Then we apply Lemma 6

$$
8\left(\frac{q 2^{k-m}+q^{2}}{\left(\alpha 2^{k-m}-1\right)^{2}}\right)^{q / 2}=8\left(\frac{q\left(1+q / 2^{k-m}\right)}{2^{k-m}\left(1-1 / 2^{k-m}\right)^{2}}\right)^{q / 2} \leq 8\left(\frac{2 q}{2^{k-m}}\right)^{q / 2} \leq \varepsilon .
$$

The above corollary establishes part (2) of Theorem 7 . The next corollary gives us a general bound which establishes part (3) of the theorem. Asymptotically it implies variants of Corollary 2 and Corollary 1, but with worse constants.

Corollary 3. For any $\varepsilon>0$ and $q:=\lceil\log (1 / \varepsilon)\rceil+3$, $a(q+1)$-wise independent hash function family $\mathcal{H}=\left\{h_{s}:\{0,1\}^{n} \rightarrow\{0,1\}^{m}\right\}_{s \in\{0,1\}^{d}}$ is $(k, 1+\alpha, \varepsilon)$ balanced for

$$
\alpha=4 \sqrt{q 2^{m-k}+\left(q 2^{m-k}\right)^{2}}=O\left(2^{m-k} \log (1 / \varepsilon)+1\right) .
$$

By setting $\delta=\varepsilon, a\left(\log \frac{1}{\delta}+4\right)$-wise independent hash function is a $(k, \delta, O(1+$ $\left.\left.2^{m-k} \log \frac{1}{\delta}\right) \delta\right)$-UExtactor.

Proof. The first part follows from Lemma 6 by noting that

$$
8\left(\frac{q 2^{k-m}+q^{2}}{\left(\alpha 2^{k-m}-1\right)^{2}}\right)^{q / 2} \leq 8\left(\frac{q 2^{k-m}+q^{2}}{\frac{1}{4}\left(\alpha 2^{k-m}\right)^{2}}\right)^{q / 2} \leq 8\left(\frac{1}{4}\right)^{q / 2} \leq \varepsilon .
$$

For the second part, we can consider two cases. If $q 2^{m-k} \leq 1$ then $\alpha \leq 4 \sqrt{2}$ and we are done. Else, $\alpha \leq 4 \sqrt{2}\left(q 2^{m-k}\right)=4 \sqrt{2}(\log (1 / \varepsilon)+3) \overline{2^{m-k}}$.

\subsection{Minimizing the Seed Length}

In both of the above constructions (Corollary 1, Corollary 2), to get an $(k, \delta, \varepsilon)$ UExtractor, we need a $O(\log (1 / \varepsilon))$-wise independent hash function $h_{s}:\{0,1\}^{n} \rightarrow$ $\{0,1\}^{m}$, which requires a seed-length $d=O(\log (1 / \varepsilon) \cdot n)$. Since in many applications, we envision $\varepsilon \approx 2^{-k}$, this gives a seed $d=O(k n)$. We should contrast this with standard extractors constructed using universal hash functions (via the leftover-hash lemma), where the seed-length is $d=n$. We now show how to optimize the seed-length of UExtractors, first to $d=O(n \log k)$ and eventually to $d=O(k \log k)$. In the full version [DPW13] of this paper, we adapt the technique of Celis et al. CRSW11. which shows how to construct hash functions with a small seed that achieve essentially optimal "max-load" (e.g., minimize the hash value with the most items inside it). We show that a lightly modified analysis can also be used to show that such hash functions are "balanced" with essentially optimal parameters.

\subsection{A Probabilistic Method Bound}

In the full version DPW13 of this paper, we give a probabilistic method argument showing the existence of unpredictability extractors with very small seed 
length $d \approx \log (1 / \delta)+\log (n-k)$ as stated in Theorem 8 below. In other words, unpredictability extractors with small entropy loss do not, in principle, require a larger seed than standard randomness extractors (with much larger entropy loss).

Theorem 8. There exists a $(k, \delta, \varepsilon)$-UExtract UExt : $\{0,1\}^{n} \times\{0,1\}^{d} \rightarrow$ $\{0,1\}^{m}$ as long as either:

$$
\begin{aligned}
\varepsilon & \geq \max \left\{2 e \delta, \quad(n-k+2) 2^{-d}+\log (e / \delta) \delta 2^{m-k}\right. \\
2 e \delta \geq \varepsilon & \geq \delta+2 \delta \sqrt{(1 / \delta)(n-k+2) 2^{-d}+\log (e / \delta) 2^{m-k}}
\end{aligned}
$$

In particular, as long as the seed-length $d \geq \log (1 / \delta)+\log (n-k+2)+3$ we get:

- In general: $\varepsilon=O\left(1+\log (1 / \delta) 2^{m-k}\right) \delta$.

- When $k=m$ and $\delta<2^{-2 e}: \varepsilon=(2+\log (1 / \delta)) \delta$.

- When $k \geq m+\log \log (e / \delta)+3: \varepsilon=2 \delta$.

\section{SRT Lower-Bound: Samplability Doesn't Improve Entropy Loss}

The 'SRT' conjecture of Dachman-Soled et al. DGKM12 states that randomness extractors need to incur a $2 \log 1 / \varepsilon$ entropy loss (difference between entropy and output length) even if we only require them to work for efficiently samplable sources. In the full version DPW13] of this paper we prove this conjecture as stated in Theorem 9 below. In fact, we show that the conjecture holds even if the extractor itself is not required to be efficient.

The efficient source for which we show a counter-example is sampled via a 4-wise independent hash function. That is, we define the source $X=h_{r}(Z)$ where $Z \leftarrow\{0,1\}^{k}$ is chosen uniformly at random and $h_{r}:\{0,1\}^{k} \rightarrow\{0,1\}^{n}$ is chosen from some 4 -wise independent hash function family. The choice of the seed $r$ will need to be fixed non-uniformly; we show that for any "candidate extractor" Ext : $\{0,1\}^{n} \times\{0,1\}^{d} \rightarrow\{0,1\}^{m}$ there is some seed $r$ such that the above efficiently sampleable $(n, k)$-source $X$ makes the statistical distance between $(\operatorname{Ext}(X ; S), S)$ and the uniform distribution at least $\approx 2^{(m-k) / 2}$.

Let Ext : $\{0,1\}^{n} \times\{0,1\}^{d} \rightarrow\{0,1\}^{m}$ be a candidate strong extractor, and let $X$ be some random variable over $\{0,1\}^{n}$. Define the distinguishability of Ext on $X$ via:

$$
\begin{aligned}
\operatorname{Dist}(X) & \stackrel{\text { def }}{=} \frac{1}{2} \sum_{s \in\{0,1\}^{d}, y \in\{0,1\}^{m}}|\operatorname{Pr}[S=s, \operatorname{Ext}(X ; s)=y]-\operatorname{Pr}[S=s, Y=y]| \\
& =\frac{1}{2^{d+1}} \sum_{s \in\{0,1\}^{d}, y \in\{0,1\}^{m}}\left|\operatorname{Pr}[\operatorname{Ext}(X, s)=y]-\frac{1}{2^{m}}\right| .
\end{aligned}
$$

where $S, Y$ are uniformly and independently distributed over $\{0,1\}^{d},\{0,1\}^{m}$ respectively. Note that $\operatorname{Dist}(X)$ is simply the statistical distance between $(S, \operatorname{Ext}(X ; S))$ and $\left(S, U_{m}\right)$ where $U_{m}$ is uniformly random $m$ bit string. 
Theorem 9. For any (possibly inefficient) function Ext : $\{0,1\}^{n} \times\{0,1\}^{d} \rightarrow$ $\{0,1\}^{m}$, any positive integer $k \geq m+2$ such that $n>3 k-m+14$, there exists a distribution $X$ with $\mathbf{H}_{\infty}(X) \geq k$, which is efficiently samplable by a poly $(n)$-size circuit, such that $\operatorname{Dist}(X) \geq 2^{(m-k) / 2-8}$.

Alternatively, for any positive $k \geq m$ such that $n>k+\log (k)+11$, there exists some distribution $X$ with $\mathbf{H}_{\infty}(X) \geq k$, which is efficiently samplable by a $\operatorname{poly}(n)$-size circuit such that $\operatorname{Dist}(X) \geq 2^{(m-k-\log (k)) / 2-9}$.

Acknowledgements. We thank Hugo Krawczyk for many enlightening discussions about the topic of this work and for suggesting that we look at the 'SRT' conjecture, which lead to the results in Section 5

\section{References}

$\left[\mathrm{BDK}^{+} 05\right]$ Boyen, X., Dodis, Y., Katz, J., Ostrovsky, R., Smith, A.: Secure remote authentication using biometric data. In: Cramer, R. (ed.) EUROCRYPT 2005. LNCS, vol. 3494, pp. 147-163. Springer, Heidelberg (2005)

$\left[\mathrm{BDK}^{+} 11\right]$ Barak, B., Dodis, Y., Krawczyk, H., Pereira, O., Pietrzak, K., Standaert, F.-X., Yu, Y.: Leftover hash lemma, revisited. In: Rogaway, P. (ed.) CRYPTO 2011. LNCS, vol. 6841, pp. 1-20. Springer, Heidelberg (2011)

[BH05] Barak, B., Halevi, S.: A model and architecture for pseudo-random generation with applications to / dev/random. In: Proceedings of the 12th ACM Conference on Computer and Communication Security, pp. 203-212 (2005)

[BR94] Bellare, M., Rompel, J.: Randomness-efficient oblivious sampling. In: 35th Annual Symposium on Foundations of Computer Science, pp. 276-287. IEEE (1994)

[BST03] Barak, B., Shaltiel, R., Tromer, E.: True random number generators secure in a changing environment. In: Walter, C.D., Koç, Ç.K., Paar, C. (eds.) CHES 2003. LNCS, vol. 2779, pp. 166-180. Springer, Heidelberg (2003)

$\left[\mathrm{CDH}^{+} 00\right]$ Canetti, R., Dodis, Y., Halevi, S., Kushilevitz, E., Sahai, A.: Exposureresilient functions and all-or-nothing transforms. In: Preneel, B. (ed.) EUROCRYPT 2000. LNCS, vol. 1807, pp. 453-469. Springer, Heidelberg (2000)

[CG89] Chor, B., Goldreich, O.: On the power of two-point based sampling. Journal of Complexity 5, 96-106 (1989)

[CRSW11] Elisa Celis, L., Reingold, O., Segev, G., Wieder, U.: Balls and bins: Smaller hash families and faster evaluation. In: Ostrovsky, R. (ed.) FOCS, pp. 599-608. IEEE (2011)

$\left[\mathrm{DGH}^{+} 04\right]$ Dodis, Y., Gennaro, R., Håstad, J., Krawczyk, H., Rabin, T.: Randomness extraction and key derivation using the $\mathrm{CBC}$, cascade and HMAC modes. In: Franklin, M. (ed.) CRYPTO 2004. LNCS, vol. 3152, pp. 494-510. Springer, Heidelberg (2004)

[DGKM12] Dachman-Soled, D., Gennaro, R., Krawczyk, H., Malkin, T.: Computational extractors and pseudorandomness. In: Cramer, R. (ed.) TCC 2012. LNCS, vol. 7194, pp. 383-403. Springer, Heidelberg (2012)

[DORS08] Dodis, Y., Ostrovsky, R., Reyzin, L., Smith, A.: Fuzzy extractors: How to generate strong keys from biometrics and other noisy data. SIAM Journal on Computing 38(1), 97-139 (2008) 
[DPW13] Dodis, Y., Pietrzak, K., Wichs, D.: Key derivation without entropy waste. Cryptology ePrint Archive, Report 2013/708 (2013), http://eprint.iacr.org/

[DRV12] Dodis, Y., Ristenpart, T., Vadhan, S.: Randomness condensers for efficiently samplable, seed-dependent sources. In: Cramer, R. (ed.) TCC 2012. LNCS, vol. 7194, pp. 618-635. Springer, Heidelberg (2012)

[DY13] Dodis, Y., Yu, Y.: Overcoming weak expectations. In: Sahai, A. (ed.) TCC 2013. LNCS, vol. 7785, pp. 1-22. Springer, Heidelberg (2013)

[GKR04] Gennaro, R., Krawczyk, H., Rabin, T.: Secure hashed diffie-hellman over non-DDH groups. In: Cachin, C., Camenisch, J.L. (eds.) EUROCRYPT 2004. LNCS, vol. 3027, pp. 361-381. Springer, Heidelberg (2004)

[HILL99] Håstad, J., Impagliazzo, R., Levin, L.A., Luby, M.: Construction of pseudorandom generator from any one-way function. SIAM Journal on Computing 28(4), 1364-1396 (1999)

[Kra10] Krawczyk, H.: Cryptographic Extraction and Key Derivation: The HKDF Scheme. In: Rabin, T. (ed.) CRYPTO 2010. LNCS, vol. 6223, pp. 631-648. Springer, Heidelberg (2010)

[KZ03] Kamp, J., Zuckerman, D.: Deterministic extractors for bit-fixing sources and exposure-resilient cryptography. In: 44th Annual Symposium on Foundations of Computer Science, pp. 92-101. IEEE, Cambridge (2003)

[NZ96] Nisan, N., Zuckerman, D.: Randomness is linear in space. Journal of Computer and System Sciences 52(1), 43-53 (1996)

[RR99] Raz, R., Reingold, O.: On recycling the randomness of states in space bounded computation. In: Proceedings of the 31st ACM Symposium on the Theory of Computing, pp. 159-168 (1999)

[RSW06] Reingold, O., Shaltiel, R., Wigderson, A.: Extracting randomness via repeated condensing. SIAM J. Comput. 35(5), 1185-1209 (2006)

[RTS00] Radhakrishnan, J., Ta-Shma, A.: Bounds for dispersers, extractors, and depth-two superconcentrators. SIAM Journal on Computing 13(1), 2-24 (2000)

[Sie89] Siegel, A.: On universal classes of fast high performance hash functions, their time-space tradeoff, and their applications (extended abstract). In: FOCS, pp. 20-25 (1989)

[TV00] Trevisan, L., Vadhan, S.: Extracting randomness from samplable distributions. In: 41st Annual Symposium on Foundations of Computer Science, pp. 32-42. IEEE, Redondo Beach (2000) 